RESEARCH ARTICLE

\title{
Nursing Students' eHealth Literacy in the U.S. and South Korea
}

\author{
Hyejin Park ${ }^{*}$ \\ Associate Professor, Florida State University College of Nursing, USA
}

*Corresponding author: Hyejin Park, PhD, RN, Associate Professor, Florida State University College of Nursing, 98 Varsity Way, P.O Box 3064310, Tallahassee, FL, 32306, USA, Tel: 850-644-5345, Fax: 850-644-7660

\begin{abstract}
Background: Online health information is a critical resource for health information seekers and, thus, nursing professionals must be able to find and distinguish the quality of health information online to support patients and caregivers. This study evaluated perceived eHealth literacy skills among nursing students in the U.S. and South Korea.

Method: eHEALS was used to assess 169 undergraduate nursing students' eHealth literacy.

Results: The study results showed that nursing students lacked confidence in using online health information to make health-related decisions and had difficulty determining whether online health resources were high or low quality. In a comparison of the two countries, we found that students in the U.S. have greater eHealth literacy.
\end{abstract}

Conclusion: Evaluation of eHealth literacy of nursing students in both countries is a fundamental data for developing curriculum to address these skills.

\section{Keywords}

eHealth literacy, Internet, Undergraduate nursing students, Online health information

\section{Introduction}

Health consumers' use of the Internet is steadily increasing throughout the world. Internet Users in the World are 4.1 billion in 2018. Asia (50.1\%) has the most Internet users of all countries followed by Europe (16.3\%), Africa (10.8\%), Latin America (10.2\%), North America (8\%) [1,2]. The Pew Research Center [3] found that $88 \%$ of Americans use the Internet. Among the Internet users, one in three American adults have sought health information from the Internet to figure out a medical condition [4]. Similarly, about $90 \%$ of Koreans in South Korea use the Internet to seek various types information and obtain advice from the Internet
[5] and $99.9 \%$ of the college students in Korea use the Internet [6]. As the Internet has become a major source of health information among health consumers, health professionals are expected to engage in online information sharing with the public and to assist healthinformation seekers with finding, understanding, and evaluating health information online [7-14].

Moreover, while the quantity of health information online is considerable, its quality widely varies. In fact, health information on some Internet sites has been found to be of low quality $[15,16]$. This inaccurate and low-quality health information can mislead patients [17] and cause them to make poor health-related decisions [18]. People may ignore important symptoms and delay or even refuse recommended health care [19]. It is, therefore, critical that patients are able to identify what health-information sites are credible and distinguish between high- and low-quality health information, so that they make health decisions using high-quality information $[20,21]$.

Nurses as health professionals need to educate patients, families, and caregivers, and they should be eHealth literate [12]. eHealth literacy is defined as the ability to seek, find, understand, and appraise health information from electronic resources and apply such knowledge to address or solve a health problem $[22,23]$. This type of literacy is an important skillset for health professionals of all kinds, so they can find reliable, valid, and high-quality health information [24] and assist patients to do the same. Tubaishat and Habiballah [12] also recognized that nurses must have eHealth literacy skills, since it is necessary for nurses to assess patients' use of eHealth information and correct any misconceptions based on incorrect

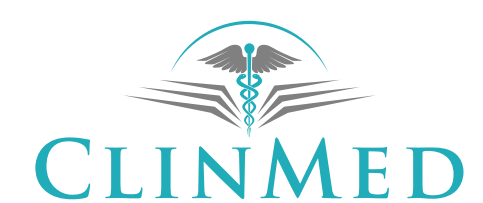

Citation: Park H (2019) Nursing Students' eHealth Literacy in the U.S. and South Korea. Int Arch Nurs Health Care 5:122. doi.org/10.23937/2469-5823/1510122

Accepted: April 24, 2019: Published: April 26, 2019

Copyright: (C) 2019 Park H, et al. This is an open-access article distributed under the terms of the Creative Commons Attribution License, which permits unrestricted use, distribution, and reproduction INTER NATIONAL LIBRARY in any medium, provided the original author and source are credited.
} 
interpretations of online information. It is important that nursing students, as future nurses, have the skills to find advanced eHealth information and to evaluate its quality $[12,15]$.

A few studies about eHealth literacy skills among undergraduate nursing students have been conducted to date $[12,15]$. One systematic review [15] found that eHealth literacy skill is lacking among college students and argued that they should be eHealth literate. Hanik and Stellefson [15] also found that students in college-level health-education programs at a large Southwestern University lack important eHealth literacy skills, especially students with less academic experience. Recently, a study [12] was conducted to assess eHealth literacy among 541 students in two universities in Jordan and fond that students lack skills related to appraisal of Internet information from low to high.

The current understanding of eHealth literacy skills could be expanded by a study of undergraduate nursing students from different countries or multiple-site colleges and universities with respondents from diverse backgrounds or cultures. Since among the college students, various factors such as type of university or culture may influence their level of eHealth literacy. Thus, this study assessed perceived level of eHealth literacy among undergraduate nursing students in different academic environment and cultures. The results from this study will enable us to identify any differences and similarities in the eHealth literacy skills (how to find, use, and critique online information) of nursing students in both countries and will serve as a fundamental dataset for the further studies related to the influencing factors of eHealth literacy from different culture, Moreover, it could eventually provide strategies for developing a culturally sensitive education tool for students.

\section{Purpose of the study}

The purpose of the study was to evaluate undergraduate nursing students' perceived eHealth literacy in the U.S. and South Korea. Its specific aims were; 1 ) To assess self-perception of the usefulness of the Internet and the importance of accessing online health information of each county; 2) To identify eight items of self-perceived eHealth literacy skills of each country; and 3) To identify differences and similarities in nursing students' level of eHealth literacy in the U.S. and South Korea.

\section{Study conceptual framework}

The relationship between data, information, knowledge, and wisdom [25] is explained as a conceptual framework. The growth of the Internet enables new ways of collecting and disseminating data, knowledge, and information. Nurses are expected to engage in disseminating data, knowledge, or online information and to assist health information seekers. Nurses need to de- velop eHealth literacy skills, such as the ability to seek, find, understand, and appraise health information from electronic resources to support reliable evidence-informed care delivery, evidence-based decision-making, continuity of care, and patient safety.

\section{Method}

\section{Design, sample, and setting}

A descriptive comparison design was used for this study and convenient sampling was used. The participants were undergraduate nursing students from two universities, one southern states within the U.S. and one in the eastern area of South Korea. In South Korea, curriculum, including nursing informatics, was incorporated into the nursing curriculum. The majority nursing students learn basic computer skills, such as Excel, PowerPoint, and Word Process, and know how to search online information but they have not had a chance to learn how to use information regarding their health-making decisions and critique this online health information. Like South Korea, nursing students in the U.S. also have basic computer skills and informatics skills. Technology Informatics Guiding Educational Reform (TIGER) recognized that basic computer skills, information literacy, and information management are three informatics competencies in all practice nurses and nursing students in 2006 . The TIGER competency collaborative has adopted the ECDL (European Computer Driving License) that is a foundation set basic computer competencies. Information literacy builds on computer literacy that includes identifying information needed for a specific purpose, locating pertinent information, evaluating the information, and applying it correctly. Information management is a process consisting of collecting data, processing the data, and presenting and communicating the processed data as information or knowledge [26]. TIGER also identified an informatics competency required for professional practice, which helped to increase the emphasis of informatics in nursing-education standards in the U.S. in 2009 [27].

An invitation email with an embedded link to a questionnaire was sent to nursing students in both universities and 169 questionnaires were completed.

\section{Measures}

An eHealth literacy tool called eHEALS [22] was used to assess eHealth literacy. The eHEALS is an eight-item, self-report questionnaire that focuses on knowledge and understanding of what health information is available on the Internet, where helpful health resources can be found, how to access this information, how to use the Internet, skills to evaluate the online health information, and the ability to discern high- and low-quality health resources on the Internet. Each item is rated on a five-point Likert scale, ranging from 1 (strongly disagree) to 5 (strongly agree). Total scores of the eHEALS 
Table 1: Characteristics of participants $(n=169)$.

\begin{tabular}{|c|c|c|c|c|}
\hline & \multicolumn{2}{|c|}{ Category } & Frequency & Percent \\
\hline \multirow[t]{2}{*}{ Age } & \multicolumn{2}{|c|}{$19-25$} & 155 & 91.7 \\
\hline & \multicolumn{2}{|c|}{$26-30$} & 14 & 8.3 \\
\hline \multirow[t]{2}{*}{ Gender } & \multicolumn{2}{|c|}{ Female } & 156 & 92.3 \\
\hline & \multicolumn{2}{|c|}{ Male } & 13 & 7.7 \\
\hline \multirow[t]{2}{*}{ Academic class } & \multicolumn{2}{|c|}{ Junior } & 44 & 26 \\
\hline & \multicolumn{2}{|c|}{ Senior } & 125 & 74 \\
\hline \multirow[t]{3}{*}{ Country } & \multicolumn{2}{|l|}{ U.S. } & 85 & 50.3 \\
\hline & \multicolumn{2}{|c|}{ South Korea } & 84 & 49.7 \\
\hline & Min & Max & Mean & SD \\
\hline $\begin{array}{l}\text { Composite eHealth } \\
\text { Literacy score }\end{array}$ & 17 & 40 & 31.95 & 5.4 \\
\hline
\end{tabular}

are summed, and range from 8 to 40 , with higher scores representing higher self-perceived eHealth literacy. An internal consistency reliability of alpha $=0.89-0.97$ with good test-retest reliability has previously been reported for this scale [22]. Two supplemental items recommended by the authors of eHEALS were included to assess perceived usefulness of the Internet for making health decisions (via a five-point Likert scale, ranging from $1=$ not useful at all to 5 = very useful) and perceived importance of being able to access health resources on the Internet (also via a five-point Likert scale, ranging from 1 $=$ not important at all to $5=$ very important). For Korean students, eHEALS was translated into Korean by two bilingual nurses at the first time and, then translated back into English by another two bilingual nurses. The first and second translated versions were agreed on.

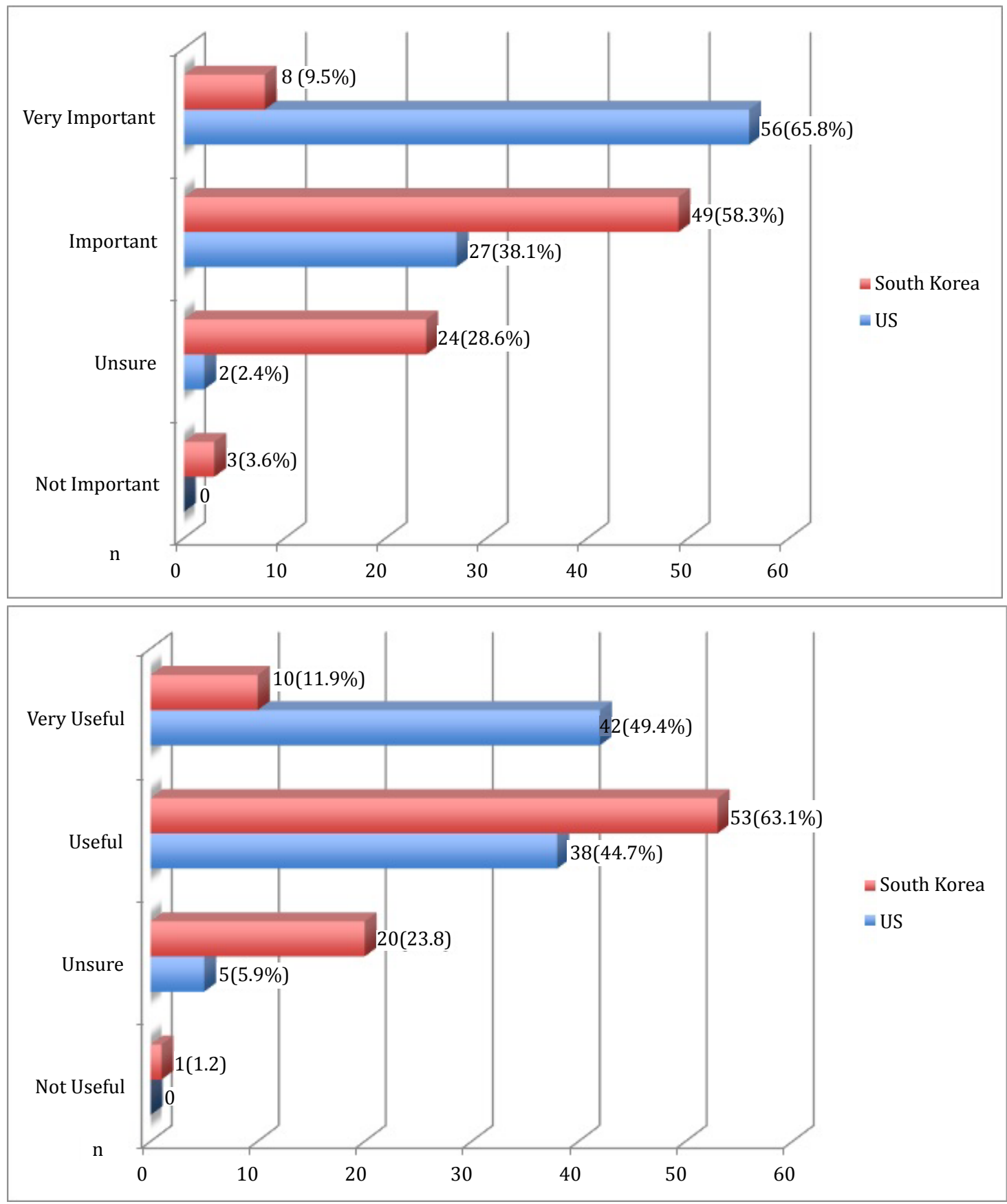

Figure 1: Usefulness of the internet and importance of accessing eHealth information in the U.S. and South Korea. 


\section{Procedures}

Permission to collect data was obtained from the nursing program directors in both countries. After Institutional Review Board (IRB) approval, researchers sent an invitation email to students enrolled in the undergraduate nursing programs in the two universities, one in the U.S. and one in South Korea. The email, which included a link to the Internet survey, included the purpose of the study, an online consent form, and the questionnaire. If a participant clicked the "agree" button, the questionnaire was automatically displayed. Data was collected over a period of one semester, of about four months. One reminder email was sent to enhance students' participation in this study.

\section{Data analysis}

To identify the mean and standard deviation (SD) of general characteristics and eHealth literacy, descriptive statistics were conducted. Independent $t$-tests were used to determine differences between the two groups' eHealth literacy.

\section{Results}

\section{Sample characteristics}

The survey was completed by 169 students in undergraduate nursing programs in two countries. The total response rate was $61 \%$ (169 out of 276 ). The great majority of participants $(n=156,92.3 \%)$ were female and fell into the age range of 19 to 25 ( $n=155,91 \%$ ). Participants from South Korea were juniors or seniors (third or fourth years): Participants from the U.S. were seniors (fourth years). The 169 respondents were evenly split from both countries (Table 1).

\section{Usefulness of the internet and importance of accessing eHealth information}

In response to questions about the usefulness of the Internet, almost $50 \%$ of participants in the U.S. reported that they believed the Internet is very useful, while only $11 \%$ of participants in South Korea perceived that the Internet is very useful tool in helping for making healthrelated decisions. Participants in the U.S. also reported that having an ability to access health information on

Table 2: eHealth literacy skills (Rating 1 to $5 ; 1$ is strongly disagree and 5 is strongly agree).

\begin{tabular}{|c|c|c|c|c|c|c|c|c|c|}
\hline \multirow[b]{2}{*}{ Items } & \multicolumn{4}{|l|}{ U.S. (n/\%) } & \multicolumn{5}{|c|}{ South Korea (n/\%) } \\
\hline & Disagree & Undecided & Agree & $\begin{array}{l}\text { Strongly } \\
\text { agree }\end{array}$ & $\begin{array}{l}\text { Strongly } \\
\text { disagree }\end{array}$ & Disagree & Undecided & Agree & $\begin{array}{l}\text { Strongly } \\
\text { agree }\end{array}$ \\
\hline $\begin{array}{l}\text { I know what health } \\
\text { resources are } \\
\text { available on the } \\
\text { Internet }\end{array}$ & 0 & $5(5.9)$ & $35(41.2)$ & $45(52.9)$ & 0 & $3(3.6)$ & $26(31)$ & $52(61.9)$ & $3(3.6)$ \\
\hline $\begin{array}{l}\text { I know where to } \\
\text { find helpful health } \\
\text { resources on the } \\
\text { Internet }\end{array}$ & 0 & $3(3.5)$ & $38(44.7)$ & $44(51.8)$ & 0 & $5(6)$ & $34(40.5)$ & $40(47.6)$ & $5(6)$ \\
\hline $\begin{array}{l}\text { I know how to } \\
\text { find helpful health } \\
\text { resources on the } \\
\text { Internet }\end{array}$ & 0 & $3(3.5)$ & $37(43.5)$ & $45(52.9)$ & 0 & $2(2.4)$ & $28(33.3)$ & $47(56)$ & $7(8.3)$ \\
\hline $\begin{array}{l}\text { I know how to use } \\
\text { the Internet to answer } \\
\text { my questions about } \\
\text { health }\end{array}$ & $1(1.2)$ & $3(3.5)$ & $30(35.3)$ & $51(60.0)$ & 0 & $4(4.8)$ & $16(19)$ & $58(69)$ & $6(7.1)$ \\
\hline $\begin{array}{l}\text { I know how to } \\
\text { use the health } \\
\text { information I find on } \\
\text { the Internet to help me }\end{array}$ & $1(1.2)$ & $2(2.4)$ & $33(38.8)$ & $49(57.6)$ & 0 & $1(1.2)$ & $14(16.7)$ & $64(76.2)$ & $5(6)$ \\
\hline $\begin{array}{l}\text { I have the skills I need } \\
\text { to evaluate the health } \\
\text { resources I find on the } \\
\text { Internet }\end{array}$ & $2(2.4)$ & $3(3.5)$ & $33(38.8)$ & $47(55.3)$ & $1(1.2)$ & $5(6)$ & $29(34.5)$ & $45(53.6)$ & $4(4.8)$ \\
\hline $\begin{array}{l}\text { I can tell high-quality } \\
\text { health resources from } \\
\text { low-quality health } \\
\text { resources on the } \\
\text { Internet }\end{array}$ & $2(2.4)$ & $4(4.7)$ & $41(48.2)$ & $38(44.7)$ & $3(3.6)$ & $17(20.2)$ & $40(47.6)$ & $23(27.4)$ & $1(1.2)$ \\
\hline $\begin{array}{l}\text { I feel confident in } \\
\text { using information from } \\
\text { the Internet to make } \\
\text { health decisions }\end{array}$ & $5(5.9)$ & $4(4.7)$ & $32(37.6)$ & $44(51.8)$ & $1(1.2)$ & $18(21.4)$ & $38(45.2)$ & $27(32.1)$ & 0 \\
\hline
\end{tabular}


the Internet is very important $(n=56,65.8 \%)$, while only eight students $(9.5 \%)$ in South Korea identified that the Internet is very important (Figure 1).

\section{Individual eight items of eHealth literacy skills}

Almost $50 \%$ of participants strongly perceived that they knew what health information is available on the Internet ( $\mathrm{n}=45,52.9 \%$ ), where to find helpful health resources ( $n=44,51.8 \%$ ), how to find helpful health resources in the Internet $(n=45,52.9 \%)$, how to use the Internet to answer their questions about health $(n=51$, $60 \%$ ), how to use the health information they found ( $\mathrm{n}$ $=49,57.6 \%)$, and how to evaluate health resources ( $n=$ $47,55.3 \%$ ), while a lower percentage reported having the ability to distinguish high-quality health resources from low-quality health resources on the Internet $(n=$ $38,44.7 \%)$. In South Korea, less than $10 \%$ of participants strongly agreed that they knew what health information is available on the Internet $(n=3,3.6 \%)$, where to find helpful health resources $(n=5,6 \%)$, how to find helpful health resources in the Internet $(n=7,8.3 \%)$, how to use the Internet to answer their questions about health ( $n=6,7.1 \%$ ), how to use the health information they found $(n=5,6 \%)$, and how to evaluate health resources ( $n=4,4.8 \%$ ), but only less than $1 \%$ of participants had the ability to tell high-from low-quality health resources ( $n=1,1.2 \%$ ). No participants were confident in using health information from the Internet to make health decisions (Table 2).

In comparing two countries, among the eight eHEALS items, independent $t$-tests were used. The biggest mean differences are "Distinguish high from low quality online health resources" followed by "feel confident in using information from the Internet to make health decisions"
(Table 3). All scores of eHealth literacy items are higher for the students in the U.S. than for students in Korea: All items have statistically significant differences at alpha level $<0.001$. The biggest mean difference between the students in the two countries was their ability to discern between high- and low-quality online health information, followed by confidence in using online health information retrieved to make health decisions. The lowest mean difference between two countries was the usefulness of the Internet to help them to make health decisions (Table 3).

\section{Discussion}

This study assessed the current eHealth literacy of undergraduate nursing students in the U.S. and South Korea using eHEALS. This study assessed the current eHealth literacy of undergraduate nursing students in the U.S. and South Korea using eHEALS. The mean eHealth literacy score was 31.95 out of 40 . This finding was higher than Tubaishat and Habiballah [12] and Hernandez-Rabanal, Vall, and Boter [28]. Tubaishat and Habiballah [12] calculated a mean score of 28.9 out of 40 from a group of 541 undergraduate students completed eHEALS. In study of Hernandez-Rabanal, Vall, and Boter [28], the mean score of perceived eHealth literacy among 298 students was 24 out of 40 . Findings from this and previous studies indicate that the mean score may vary depending on demographic characteristics such as age, gender, academic experiences such as health-related majors or non-health related majors, and Internet-use experiences that may influence eHealth literacy $[14,29]$. Tennant, et al.'s [29] study showed that people with a younger age, more education, and more use of electronic devices have greater eHealth literacy

Table 3: Comparison of eHealth literacy between the U.S. and South Korea undergraduate nursing students (Rating 1 to 5,1 is strongly disagree and 5 is strongly agree).

\begin{tabular}{|c|c|c|c|c|c|}
\hline & U.S. & South Korea & & & \\
\hline Items & $M(S D)$ & $M(S D)$ & $t$ & $d f$ & $P$ \\
\hline $\begin{array}{l}\text { How useful do you feel the Internet is in helping you in making } \\
\text { decisions about your health? }\end{array}$ & $4.47(0.54)$ & $3.86(0.62)$ & 6.79 & 167 & $0.000^{*}$ \\
\hline $\begin{array}{l}\text { How important is it for you to be able to access health resources on } \\
\text { the Internet? }\end{array}$ & $4.64(0.53)$ & $3.74(0.67)$ & 9.57 & 167 & $0.000^{*}$ \\
\hline I know what health resources are available on the Internet & $4.48(0.59)$ & $3.65(0.61)$ & 8.95 & 167 & $0.000^{*}$ \\
\hline I know where to find helpful health resources on the Internet & $4.48(0.56)$ & $3.54(0.70)$ & 9.63 & 167 & $0.000^{*}$ \\
\hline I know how to find helpful health resources on the Internet & $4.49(0.57)$ & $3.70(0.65)$ & 8.39 & 167 & $0.000^{*}$ \\
\hline I know how to use the Internet to answer my questions about health & $4.54(0.62)$ & $3.79(0.64)$ & 7.73 & 167 & $0.000^{*}$ \\
\hline $\begin{array}{l}\text { I know how to use the health information I find on the Internet to } \\
\text { help me }\end{array}$ & $4.53(0.60)$ & $3.87(0.51)$ & 7.63 & 167 & $0.000^{*}$ \\
\hline $\begin{array}{l}\text { I have the skills I need to evaluate the health resources I find on the } \\
\text { Internet }\end{array}$ & $4.45(0.71)$ & $3.55(0.73)$ & 8.06 & 167 & $0.000^{*}$ \\
\hline $\begin{array}{l}\text { I can tell high-quality health resources from low-quality health } \\
\text { resources on the Internet }\end{array}$ & $4.35(0.685)$ & $3.02(0.82)$ & 11.43 & 167 & $0.000^{*}$ \\
\hline $\begin{array}{l}\text { I feel confident in using information from the Internet to make health } \\
\text { decisions }\end{array}$ & $4.34(0.825)$ & $3.08(0.76)$ & 10.28 & 167 & $0.000^{*}$ \\
\hline
\end{tabular}

Note: SD: Standard Deviation; M: Mean; ${ }^{*} p$ value $<0.001$. 
than those who do not. Another study [14] identified that students who majored in medicine have better eHealth literacy. Regarding perceived usefulness and importance, participants agreed that the Internet is useful in helping them make health-related decisions. They also agreed that it is important to have access online health resources. Tubaishat \& Habiballah [12] also found that, among 541 nursing students, $70 \%$ perceived the Internet as useful and $64 \%$ agreed that accessing online health resources was important.

In this study, participants' scores regarding how to use the online health information that they found and how to use the Internet to answer questions about health were higher than their scores on how or where to find helpful online health resources, and having knowledge about available health resources on the Internet in both countries. One possible reason is that they do not have enough knowledge about how or where to find helpful online health information.

Participants lacked confidence in using online information to make their health-related decisions in this study. Specifically, participants felt that they had difficulty determining whether health resources on the Internet were of high or low quality. Determining the quality of health resources is an important skill for nursing students. In a systematic review, Eysenbach and colleagues [30] found that $70 \%$ of 170 articles concluded that quality is a problem on the web. Another study by Baker, et al. [31] identified that most websites are poor quality to facilitate health-related decision making. Low-quality health information sites can influence health consumers' health outcomes and health-related decisions [32]. Therefore, health consumers, including both patients and caregivers, should be taught and assisted by health professionals, such as nurses, to evaluate and apply high-quality online health information for the effective management of their health problems and health decisions [33]. This skill is a competency that should be required for nurses, and, so, needs to be taught to students in nursing programs to provide safe and comprehensive care. Park and Kim [34] also suggested that college students should be required to learn about eHealth literacy in curriculum.

In comparing eHealth literacy in students from both countries, students in the U.S. had higher perceived eHealth literacy scores for all eight items than the students in South Korea. All perceived eHealth items had statistically significant results. The high- and lowquality online health information is the biggest mean difference among the eight items. There are many possible reasons for this difference, including different secondary school and nursing curricula in the two countries and differing health-consumer demands. Among college students, various individual factors, such as health status, major, and degree of health concern, are factors that influence their level of eHealth literacy [23], but further qualitative studies are needed to explore these influencing factors. From the results of this study, areas of weakness in eHealth literacy in the nursing curriculum have been identified. For example, one weakness shown in this study was the ability to discern the quality of information: This is a skillset that Korean undergraduate students, in particular, need improvement. With this new finding, educators may now be more aware of the weakness and address these weaknesses when developing or revising curriculum. The study's results will also support development of learning activities to assist future nurses to acquire the competencies in these foundational nursing skills.

There are some limitations of the study. First, the sample may not be representative of the population, since students are from only one university in each country and it was small sample size. It is also necessary to gather information about students' general characteristics beyond age, gender year, academic year, and countries: For example, Internet skills, computer skills, any experiences in terms of health informatics or eHealth literacy, or computer-skill education. This limitation may influence the comparison of the two countries' eHealth literacy results. Also, participants in the U.S. were all senior-year students, while participants in South Korea were both juniors and seniors: This difference in experience may be enough to influence students' eHealth literacy proficiency. Self-reported eHealth literacy may also affect to the study results because response bias is a common problem in selfreported data. The participants' responses in this study may do not reflect the actual eHealth literacy skills.

\section{Conclusions}

With the increasing use of the Internet, health professionals face challenges and opportunities. Nursing professionals, in particular, need to develop a new attitude toward health information on the Internet and become proficient in skills, such as eHealth literacy to better assist patients and caregivers to access and use high-quality online health information. Evaluating undergraduate nursing students' level of eHealth literacy is the first step to developing their eHealth literacy through changes in the nursing curriculum and other methods. In this study, level of eHealth literacy of nursing students was identified. Among the 8 items of eHealth literacy scale, the ability to discern the quality of online information is needed to improve in both countries. With this finding, educators may now be more address the weakness when developing or revising nursing curriculum. The study's results will also support future nurses to developing this skill during their training, future nurses will become eHealth literate - a requisite competence for all future health professionals.

\section{References}


1. Internet World Stats (2019) Internet users in the world by regions - March 2019.

2. John S (2018) Internet stats \& facts for 2019.

3. Aaron S (2017) Record shares of Americans now own smartphones, have home broadband. Pew Research Center.

4. Susannah F, Maeve D (2013) Health online 2013. Pew Research Center.

5. The Statistics Portal (2016) Internet usage in South Korea - Statistics and facts.

6. Kim JY, Cho CH, Jang SJ, Youn EJ (2015) Survey on the internet usage. Research Report. Korea Internet \& Security Agency, Seoul.

7. Britt RK, Collins WB, Wilson K, Linnemeier G, Englebert AM (2017) eHealth literacy and health behaviors affecting modern college students: A pilot study of issues identified by the American College Health Association. J Med Internet Res 19: e392.

8. Chang SJ, Yang E, Ryu H, Kim HJ, Yoon JY (2018) Crosscultural adaptation and validation of the eHealth literacy scale in Korea. Korean J Adult Nurs 30: 504-515.

9. Chung S, Park BK, Nahm ES (2018) The Korean eHealth Literacy Scale (K-eHEALS): Reliability and validity testing in younger adults recruited online. J Med Internet Res 20: e138.

10. Kim SH, Son YJ (2017) Relationships between eHealth literacy and health behaviors in Korean adults. Comput Inform Nurs 35: 84-90.

11. Paige SR, Stellefson M, Chaney BH, Chaney JD, Alber JM, et al. (2017) Examining the relationship between online social capital and eHealth literacy: Implications for instagram use for chronic disease prevention among college students. Am J Health Educ 48: 264-277.

12. Tubaishat $A$, Habiballah $L$ (2016) eHealth literacy among undergraduate nursing students. Nurse Educ Today 42: 47-52.

13. MacCabe K, Hull D (2014) How patients use online sources for information. Nursing Times 110: 24-25.

14. Yang SC, Luo YF, Chiang CH (2017) The associations among individual factors, eHealth literacy, and healthpromoting lifestyles among college students. J Med Internet Res 19: e15.

15. Stellefson M, Hanik B, Chaney B, Chaney D, Tennant B, et al. (2011) eHealth literacy among college students: A systematic review with implications for eHealth education. J Med Internet Res 13: e102.

16. Kaicker J, Dang W, Mondal T (2013) Assessing the quality and reliability of health information on ERCP using the DISCERN instrument. Health Care Current Reviews 1: 104.

17. Tabitha T, Gillian B, Cvetan T (2014) Health information on the internet. Can Fam Physician 60: 407-408.

18. Office of disease prevention and health promotion (2000) Health communication. In: Health people 2000. ( $2^{\text {nd }}$ edn), Department of Health and Human services, Washington DC, 11-25.
19. Kitchens B, Harle CA, Li S (2014) Quality of health-related online search results. Decision Support Systems 57: 454462.

20. Norman CD, Skinner HA (2006a) eHealth literacy: Essential skills for consumer health in a networked world. J Med Internet Res 8: e9.

21. Diviani N, Meppelink CS (2017) The impact of recommendations and warnings on the quality evaluation of health websites: An online experiment. Computers in Human Behavior 71: 122-129.

22. Norman CD, Skinner HA (2006b) eHEALS: The eHealth literacy scale. J Med Internet Res 8: e27.

23. Hsu W, Chiang C, Yang S (2014) The effect of individual factors on health behaviors among college students: The mediating effects of eHealth literacy. J Med Internet Res 16: e287.

24. Ivanitskaya L, Neter E, Matingwina T, Stephens M (2011) eHealth literacy among college students: A systematic review with implications for eHealth education. JMIR 13: e102.

25. Cooper P (2017) Data, information, knowledge and wisdom. Anaesthesia \& Intensive Care Medicine 18: 55-56.

26. HIMSS (2017) The evolution of TIGER competencies and informatics resources.

27. O'Connor S, Hubner U, Shaw T, Blake R, Ball M (2017) Time for TIGER to ROAR! Technology informatics guiding education reform. Nurse Educ Today 58: 78-81.

28. Hernandez-Rabanal C, Vall A, Boter C (2018) Training, the key to improving eHealth literacy of upper secondary school students. Gac Sanit 32: 48-53.

29. Tennant B, Stellefson M, Dodd V, Chaney B, Chaney D, et al. (2015) eHealth Literacy and Web 2.0 health information seeking behaviors among baby boomers and older adults. J Med Internet Res 17: e70.

30. Eysenbach G, Powell J, Kuss O, Sa ER (2002) Empirical studies assessing the quality of health information for consumers on the world wide web: A systematic review. JAMA 287: 2691-2700.

31. Baker D, Marshall J, Lee M, Jones G, Brown S, et al. (2017) P443 Systematic review of internet decision making resources for patients considering surgery for ulcerative colitis. Journal of Crohn's and Colitis 11: S302-S303.

32. Xiao N, Sharman R, Rao HR, Upadhyaya S (2014) Factors influencing online health information search: An empirical analysis of a national cancer-related survey. Decision Support Systems 57: 417-427.

33. Park H, Cormier E, Gordon G, Baeg JH (2016) Identifying health consumers' eHealth literacy to decrease disparities in accessing eHealth information. Comput Inform Nurs 34: 71-76.

34. Park JW, Kim M (2017) A comparison study of e-Health literacy and self-care agency between nursing students and non-health department women college students. J Korean Acad Nurs Adm 23: 439-449. 